\title{
La finance comportementale : Revue de littérature
}

\author{
My Hicham SNINEH ${ }^{1}$ et Hicham MESK $^{2}$ \\ ${ }^{1}$ Faculté des Sciences Juridiques, Économiques et Sociales Ain Chock, Université Hassan II, Casablanca, Maroc \\ Laboratoire : Business Intelligence, Gouvernance des Organisations, Finance et Criminalité Financière \\ ${ }^{2}$ Faculté des Sciences Juridiques, Économiques et Sociales Ain Chock, Université Hassan II, Casablanca, Maroc \\ Laboratoire : Business Intelligence, Gouvernance des Organisations, Finance et Criminalité Financière
}

\begin{abstract}
Résumé : La finance classique ou standard ayant affiché certaines limites, notamment quant à l'explication voire la prédiction des dysfonctionnements des marchés boursiers, la finance comportementale propose un angle de vue supplémentaire en intégrant la psychologie des investisseurs comme complément d'analyse de tels évènements. Les travaux de recherche en la matière explicitent certes ce nouveau paradigme, mais se consacrent rarement à l'ensemble de ses aspects. C'est pourquoi, sans prétendre l'exhaustivité, l'objectif de ce papier est de mettre à la disposition des chercheurs en finance une revue de littérature touchant un maximum d'aspects, notamment la volatilité, la rationalité, ainsi que les biais et heuristiques. Nous adoptons alors une approche comparative pour mettre en évidence les apports de la finance comportementale remettant en cause la double hypothèse de rationalité et d'efficience qui fondent la finance classique.
\end{abstract}

Mots-clés : Rationalité ; efficience ; risque fondamental ; volatilité ; biais comportementaux, heuristiques.

\section{Introduction}

La finance classique en général et la théorie sur l'efficience des marchés en particulier demeurent jusqu'à nos jours de principaux fondamentaux de la finance. Leur apport en la matière n'est nullement à démontrer, non pas parce qu'en réalité le fonctionnement des marchés financiers ne souffre d'aucune anomalie, mais parce que les modèles classiques résument une approche scientifiquement correcte, dont les fondements sont solidement bâtis, aussi bien mathématiquement qu'empiriquement.

Nous parlons tout de même d'auteurs de grande renommée dont les compétences sont reconnues et dont les traces scientifiques laissées sont indélébiles.

Cependant, cela n'exclut pas que cette discipline soit perfectible sur plusieurs que la finance comportementale a mis en évidence.

\section{Lacunes de la finance classique vues par le courant comportementaliste}

La finance classique consiste à comprendre les marchés financiers à travers des modèles dans lesquels les agents sont supposés être parfaitement rationnels ou raisonnables. Cette rationalité a un double sens. D'abord, en interceptant de nouvelles informations, les agents mettent à jour leurs croyances de manière correcte. Ensuite, ceci étant, ces agents sont capables de prendre des décisions fondées sur des 
calculs statistiques et probabilistes complexes, compatibles avec la notion d'utilité. L'usage des mathématiques, combiné au recours à l'outil informatique, trouve à ce niveau tout son sens actuellement. (Thaler [2005]).

\subsection{Volatilité et valeur fondamentale}

Le fait d'investir en Bourse consiste à mobiliser la partie non consommée du revenu dans un domaine à risque en acquérant un titre financier dont la rentabilité future est généralement incertaine. Cette décision exige donc le calcul d'une valeur qui dépend largement du risque qui l'entoure.

\subsubsection{Volatilité excessive des valeurs}

$\mathrm{Au}$ regard de la finance classique, l'investisseur qui achète une action, étant rationnel et averse au risque, essaie de maximiser ses revenus en actualisant ses dividendes futurs moyennant un taux de rentabilité espéré acceptable. Cette technique résume le modèle d'équilibre des actifs financiers (MEDAF) développé par Sharpe [1964].

Pour rester attentif à la déviation du cours de son action par rapport à sa valeur fondamentale, l'investisseur en question considère que celle-ci reflète toutes les informations disponibles. Justement, c'est ce que suppose l'hypothèse de l'efficience informationnelle telle qu'elle est conçue par Fama [1965]. L'auteur classe ce caractère d'efficience en trois types imbriqués, selon le niveau de disponibilité des informations, comme suit :

Le premier type, dit efficience de forme faible, stipule que les cours reflètent correctement toutes les informations du marché disponibles. Dans ce cas, les valeurs passées d'un titre expliquent son rendement actuel et y sont donc représentées.

Selon le deuxième type, dit efficience de forme semi-forte, le prix d'un titre incorpore non seulement les informations définies dans le premier type, mais également l'ensemble des informations publiques. Cependant, l'usage de ces dernières ne rapporte aucun rendement supplémentaire.

Le troisième type, dit efficience de forme forte, stipule que le prix d'un actif incorpore toutes les informations, publiques et privées, car elles sont accessibles à tous. C'est pourquoi, aucun profit particulier ne peut être tiré par un investisseur particulier sur ce type de marché.

La finance classique suppose également que, les marchés étant efficients, le cours effectif d'un titre financier est l'image parfaite de sa valeur fondamentale. Nous pouvons trouver déjà une première origine de cette hypothèse d'efficience chez Adam Smith qui confirme l'existence d'une certaine "main invisible" (Smith [1759]) permettant au marché de s'autoréguler continuellement et aux valeurs de fluctuer sans trop dévier.

Illustrons ce double postulat et considérons un marché efficient où opèrent des agents rationnels. A le voir de plus près, ce marché n'admet pas de frictions. Percevant et assimilant totalement les informations, ces agents n'ont qu'à actualiser les rendements espérés futurs, moyennant un taux d'intérêt acceptable sur le marché. Autrement dit, aucune autre stratégie d'investissement n'apporte plus que la moyenne des gains que le risque y afférent justifie.

La théorie financière classique demeure dans ce sens une référence. Mais, toujours est-il que la question autour de l'incapacité de ses modèles à prévenir la survenance de grandes déviations des prix du marché pose problème.

Aussi simple que cela puisse paraître, la finance classique pourrait être convaincante si les prédictions qu'elle émet se confirment toujours dans la réalité. Or, ce n'est pas le cas. Les rendements des titres objet de pratiques spéculatives par exemple sont quasiment imprédictibles (Shiller [1984]). 
Avec la succession des dysfonctionnements qui affectent les marchés boursiers à travers le monde, il devient de plus en plus clair que le paradigme de la finance classique n'aide ni à comprendre facilement tous les comportements individuels ou collectifs des investisseurs, ni -dans une plus grande échelle- à anticiper le déclenchement des crises boursières.

Plus encore, quand une crise financière s'installe, ce défaut s'étend aussi à l'apport d'éléments d'explication, naturellement attendus par les acteurs du marché dans pareilles situations.

Les critiques à l'encontre de la finance classique sont émises sur plusieurs aspects et depuis bien longtemps, notamment par Friedman et al. [1948] quant aux choix risqués, par Allais [1953] qui a introduit la notion de réalité psychologique dans l'appréhension des situations à risques, ainsi que par Ellsberg [1961], pour ne citer que ces dates et ces auteurs.

Justement, la volatilité excessive des prix, à l'origine de plusieurs types de dysfonctionnements sur le marché, n'est tout simplement pas prédite par la technique d'actualisation des gains futurs, aussi pertinente et couramment utilisée qu'elle soit.

Déjà, Shiller [1981] qui avait pertinemment prédit la formation de la bulle internet lors des années 1990 et la bulle spéculative relative à l'immobilier au milieu des années 2000, avait déjà démontré que les cours des actions sont excessivement volatiles comparés aux dividendes.

L'éloignement parfois très remarquable du cours d'un titre par rapport à sa valeur fondamentale montre que les deux ne sont pas forcément en corrélation positive, comme le démontrent Shiller [1981], ainsi que LeRoy et al. [1981] qui ont travaillé sur les valeurs de l'indice Standard \& Poors 500 au Wall Street. Toujours selon Shiller [1981], la volatilité exagérée montre que le prix ne reflète pas les fondamentaux de la valeur en question.

Outre la valeur fondamentale issue généralement d'un calcul probabiliste (Orléan [2004]), chaque action se caractérise par un risque intrinsèque que Markowitz [1952] propose de maitriser en diversifiant la composition du portefeuille. Ledit risque est au fait la volatilité.

De Bondt et al. [1985] émettent une première critique sur l'efficience du marché dite "Inversion de long terme" en travaillant sur l'historique des rentabilités des actifs financiers. En effet, ils démontrent qu'empiriquement les actifs gagnants dans un passé récent peuvent devenir perdants à long terme ; et inversement.

Par ailleurs, selon l'efficience du marché, l'information est supposée gratuite parce que les prix la contiennent. Or, en réalité, chercher l'information a paradoxalement un coût (Stiglitz et al. [1980]).

Shiller [1984] a également eu l'idée de comparer l'historique des valeurs d'un certain nombre de titres sur le marché à celui de leurs valeurs intrinsèques. Il confirme que les premières fluctuent beaucoup plus que ne le font les secondes.

Des essais d'analyse de la fluctuation des prix ont été aussi menés par Grossman et al. [1981], par Shiller [2003], par Lardic et al. [2006], par Pollin [2007], puis par Hommes et al. [2008]. Tous ces auteurs attribuent la volatilité exagérée aux pratiques rentières qui mènent souvent vers la formation des bulles spéculatives. La volatilité excessive peut s'expliquer aussi par la présence de phénomènes de mode (Lardic et al. [2006]).

Ellsberg [1961] démontre de son côté que les agents ne sont pas averses au risque uniquement, mais aussi à l'ambiguiité qui découle de l'incertitude autour des probabilités. Leurs réactions peuvent même être agressives quand ils n'arrivent pas à accéder à une information censée être disponible. 
Dans ce sens, la finance classique semble omettre un détail qui fait tout de même la différence. Elle considère la valeur d'un actif estimée par un agent donné comme une information objective et exogène (Orléan [2008]), au-delà de toutes croyances ou préférences que cet agent puisse développer.

De son côté, la finance comportementale, certes, écarte le postulat de la rationalité parfaite et s'accorde à confirmer l'existence de frictions et d'un impact de l'environnement institutionnel sur le marché (De Bondt et al. [2008]). Elle ne nie par contre pas que le titre financier ait une valeur objective calculable (Orléan [2004]) qui oriente l'agent vers telle ou telle option.

En outre, en plus de la valeur objective, un actif financier aurait une valeur subjective issue des croyances, des préférences et des comportements parfois biaisés de l'agent qui s'y intéresse (Orléan [2004]).

Dans ce même sens, Léger [2008] assimile le titre en Bourse à un produit d'image immatérielle dont la valeur incorpore, en plus du couple risque-rentabilité, les aspects de notoriété, de proximité, ou encore de confiance. Un simple fait divers en faveur ou à l'encontre de la société en question impactera le cours de son titre coté.

Parallèlement, le nom de l'entreprise cotée peut avoir une influence sur les préférences des investisseurs et donc sur les cours. En effet, selon l'effet dit "dotcom", les entreprises qui changent leurs noms de domaine vers ".com" voient les cours de leurs valeurs cotées augmenter (Cooper et al. [2001]).

Ces exemples, qui ne sont pas exhaustifs, démontrent a priori que la finance classique ne considère pas la volatilité excessive comme une réalité récurrente sur le marché. C'est ainsi que l'intérêt doive être orienté vers ce risque de déviation, lié ne serait-ce qu'à la valeur fondamentale elle-même.

\subsubsection{Risque fondamental ignoré}

Bien que la finance classique ne nie pas l'existence du risque, elle semble en ignorer certaines facettes. Nous en présentons les principales comme suit.

Commençons par un fait particulier : l'introduction en Bourse. Un titre introduit dans un indice voit en principe sa valeur augmenter à court terme (Shleifer [1986]). Le prix initial proposé est généralement attractif pour encourager un maximum d'investisseurs à l'acquérir et réussir ainsi l'opération d'introduction.

Par conséquent, opter pour des titres sur le marché primaire rapporterait aux preneurs des rendements supérieurs à court et à moyen termes (Ibbotson [1975]). Or, aussi simple soit-il, ce mécanisme ne peut curieusement pas être expliqué par les modèles moyenne-variance.

Considérons maintenant l'exemple d'une valeur mobilière A sur le marché secondaire. Évidemment, rien n'empêche que ce titre, acquis par des agents rationnels dans l'optique d'une revente ultérieure revalorisée, puisse chuter suite à des contre-performances de l'entreprise (Thaler [2005]). C'est ce que l'on appelle risque fondamental, ou risque lié à la valeur fondamentale.

Si les investisseurs en question optent pour l'acquisition d'un titre équivalent B (de remplacement) pour sécuriser leurs portefeuilles, cela ne sera pas nécessairement performant à la même époque. Ce mécanisme reste vulnérable notamment si l'entreprise B opère dans la même branche d'activité parce qu'elle demeure exposée et sensible à toute nouvelle défavorable concernant cette branche. Ceci risque de ne pas combler entièrement le risque fondamental relatif à la valeur A. (Thaler [2005]).

En outre, acquérir des titres de remplacement est assorti de frais, ce qui représente un coût de plus. Ces frais sous forme d'honoraires ou de commissions sont parfois très chers (D'Avolio [2002]). Il se peut même que les investisseurs soient financièrement disposés à payer sans pour autant trouver des titres à 
prendre. C'est le cas par exemple lorsque la réglementation en vigueur ne permet pas de réaliser des transactions similaires (Merton [1987]).

Par ailleurs, exploiter lucrativement une déviation du prix d'un titre par rapport à sa valeur fondamentale exige une bonne compréhension de la transaction. Un processus d'apprentissage intellectuel et technique est donc nécessaire et a bien sûr un coût (Merton [1987]).

En somme, investir sur le marché financier pose une question sur la définition de la valeur de référence. En effet, celle-ci peut être déterminée à travers l'historique des dernières valeurs cotées (Grinblatt et al. [2000]). Mais, comme très souvent, les agents utilisent le taux de rentabilité de l'actif sans risque pour capitaliser leurs fonds (Barberis et al. [2001]). Le résultat obtenu est pour eux la valeur de référence.

Ceci étant pour la valeur des titres, qu'en est-il alors pour la rationalité de l'agent ?

\subsection{Réalité de la rationalité}

Étant un attribut qualitatif, la rationalité demeure un concept compliqué à cerner. Il s'agit également d'un idéal qui a été formalisé mathématiquement dans le cadre de la finance classique.

Mais, il a fallu plusieurs années de recherche hors sciences exactes, notamment en psychologie, pour prouver que la rationalité est quasiment impossible à vérifier dans tous les comportements humains.

\subsubsection{Inexistence de la rationalité parfaite}

Pour faire des prévisions, les modèles comportementaux ont besoin d'abord de spécifier la forme de la non rationalité parfaite des agents. Il s'agit ici de la question centrale autour de la rationalité, que nous pouvons formuler comme suit: Comment les investisseurs n'observent-t-ils pas correctement les fondamentaux et dévient de l'utilité ?

Afin de répondre à ce questionnement, les modèles proposés par le courant comportementaliste s'appuient sur des expériences menées par des spécialistes en psychologie cognitive sur les biais pouvant provenir des croyances que les agents développent ou des préférences qu'ils forment. Justement, la psychologie cognitive a transformé les théories économiques de la décision (Schmidt [2006]).

Dans ce sens, Stracca [2004] recommande d'explorer davantage la finance comportementale en vue de mettre la rationalité des agents sous la lumière.

Contrairement à la théorie financière classique, la finance comportementale stipule que les prix des titres peuvent dévier de leurs valeurs fondamentales, et que cette déviation émane de la présence d'agents non complètement rationnels. Friedman [1953] admet l'existence de ces derniers, mais rejette le reste de ce postulat en confirmant que ladite déviation créée est aussitôt effacée par des agents rationnels. Ces derniers jouent ainsi le rôle d'arbitragistes lorsqu'ils mènent des actions d'achats ou de vente en guise de rééquilibrage (Szyszka [2013]).

Le concept d'arbitrage consiste à effectuer une opération d'achat d'un titre simultanément à une opération de vente d'un autre titre dont les attributs sont similaires au premier; ou inversement (Sharpe et al. [1990]). Les deux titres sont supposés substituables. C'est pourquoi un marché sans arbitragistes verra le volume de transactions en son sein diminuer, même avec la disponibilité de toute l'information et avec toute la capacité de prédiction et d'analyse chez les agents rationnels (Miller et al. [1961]).

Si le titre vendu est plus cher que celui acheté, sa valeur diminuera naturellement car son offre agrégée a augmenté. Inversement, le titre acheté devient moins disponible et donc désiré, d'autant qu'il coûte 
moins cher. C'est pourquoi sa demande agrégée vue à la hausse fera augmenter son cours jusqu'à rejoindre celui du titre vendu (Sharpe et al. [1990]).

Pour illustrer ce raisonnement, Friedman [1953] considère un groupe d'investisseurs non parfaitement rationnels. Il suppose qu'ils sont pessimistes quant aux perspectives de la société Ford cotée à $20 \$$, et cèdent les actions qu'ils y détiennent, ramenant ainsi leur valeur fondamentale à $15 \$$. Pressentant en cela une opportunité lucrative dans le futur, non seulement les arbitragistes achèteront à ce dernier prix les actions cédées, mais ils mettront parallèlement en vente une valeur mobilière alternative ayant une rentabilité similaire, comme celle de la société General Motors. La pression subie par l'action Ford ramènera son prix à sa valeur fondamentale initiale.

Pour les comportementalistes, l'argument de Friedman est contestable sur ce point de vue car l'opération d'arbitrage présente plusieurs limites (Shleifer et al. [1997]). A l'occasion d'une grande déviation causée par les investisseurs non parfaitement rationnels, les stratégies menées par leurs homologues rationnels pour la corriger peuvent être risquées et coûteuses, et ne représentent donc pas une opportunité attractive.

En plus, si le marché est efficient et les cours évalués y sont justes, il est normal de confirmer qu'aucune opération d'arbitrage n'est lucrative. Mais l'inverse n'est pas vrai. S'il n'existe pas de possibilité d'arbitrage, cela ne signifie pas que le marché est efficient. (Barberis et al. [2002])

Enfin, à part les cas exceptionnels des actions jumelles, affiliées à différentes entreprises mais qui donnent droit à des dividendes identiques (Lamont et al. [2003]), la loi du prix unique (Berk et al. [2017]) veut que les valeurs cotées, parfaitement identiques et ayant les mêmes perspectives, doivent aussi avoir des prix identiques (Lamont et al. [2003]). Sinon, tout investisseur peut acheter les valeurs moins chères et vendre les plus chères, sans avoir à être rationnel ou connaisseur.

Cette confrontation de pensées est mise en évidence par l'identification de plusieurs types de risques. Ces derniers prouvent l'incapacité des gestionnaires de portefeuilles, même suffisamment avertis, à faire face aux aléas du marché (Rubinstein [2000]).

\subsubsection{Risque lié à la non rationalité}

Il s'agit là d'un postulat introduit par De Long et al. [1990] et étudié avec davantage de profondeur par Shleifer et al. [1997]. Selon ces auteurs, le risque exploité par les arbitragistes peut s'aggraver à court terme.

Pour l'exemple cité plus haut, même si la valeur General Motors parait pouvoir représenter un bon titre de remplacement vis-à-vis de celui de Ford, les agents rationnels demeurent confrontés au risque que les investisseurs non rationnels, à l'origine de la dépréciation de la valeur Ford, deviennent plus pessimistes et la font baisser davantage.

Ceci étant, les agents rationnels peuvent par conséquent être contraints de se débarrasser du titre Ford plus tôt que prévu, et subir les éventuelles pertes qui en découlent. Et ce, pour une simple raison développée par Shleifer et al. [1997] : les agents rationnels gèrent éventuellement des capitaux ou des portefeuilles qui ne sont pas nécessairement les leurs (Shleifer [2000]). Parfois, ils peuvent juste vouloir faire plaisir aux actionnaires (Sen [1991]).

Cette configuration n'est évidemment pas sans conséquences. Les investisseurs dépourvus de connaissances techniques suffisantes pour comprendre et apprécier les stratégies des agents rationnels, évalueront les titres tout simplement selon leurs rendements actuels. Si ces derniers sont faibles ou négatifs, les investisseurs auront tendance à retirer leurs fonds. 
Le problème s'aggrave si les valeurs mobilières sont des titres de créance. En présence d'une baisse des rendements, les créanciers concernés réclameront le remboursement, déclenchant de nouveau une liquidation prématurée.

Par ailleurs, les agents ne peuvent pas toujours constituer des portefeuilles diversifiés de manière optimale (Shleifer [2000]). À titre d'exemple, cinq actifs au plus constituent les portefeuilles dans la réalité (Goetzman et al. [2008]). C'est pourquoi les agents font souvent dévier leurs investissements des niveaux efficients tels qu'ils sont conçus par Markowitz (Shefrin et al. [2000]).

Suite à une diversification "naïve" (Benartzi et al. [2001]) ignorant le couple rendement-risque, lesdits portefeuilles peuvent comporter majoritairement des actifs positivement asymétriques, c'est-à-dire dont les attributs sont similaires à ceux d'un jeu de loterie (Barberis et al. [2001]).

Une autre explication de la violation du cadre de la rationalité stipule que les agents (salariés) optent souvent pour les titres de l'entreprise pour laquelle ils travaillent. Pour eux, il s'agit d'un investissement moins risqué puisqu'ils estiment bien connaître leurs portefeuilles (Huberman [2001]). Par conséquent, ils peuvent y investir de manière excessive (Benartzi [2001]).

Ainsi, les comportements de ces agents tendent à déformer les pondérations objectives liées au calcul probabiliste risque/gain potentiel (Kahneman et al. [1982]).

Pour illustrer ce phénomène, nous reprenons le travail empirique mené par Kahneman et al. [1979]. L'expérimentation en question consiste à proposer à une cohorte de participants deux configurations où dans chacune d'elles ils sont amenés à choisir entre deux loteries, comme décrit ci-après :

Configuration 1:

\begin{tabular}{|l|l|}
\hline \multicolumn{1}{|c|}{ Loterie $\mathbf{A}$, à risques } & \multicolumn{1}{c|}{ Loterie $\mathbf{B}$, sans risque } \\
\hline - Gain : $2500 \$$ avec $33 \%$ de chances & - Gain $: 2400 \$$ avec $100 \%$ de chances \\
- Gain : $2400 \$$ avec $66 \%$ de chances \\
- Gain : $\quad 0 \$$ avec $1 \%$ de chances
\end{tabular}

Configuration 2 :

\begin{tabular}{|l|l|}
\hline \multicolumn{1}{|c|}{ Loterie C, à risques } & \multicolumn{1}{c|}{ Loterie D, sans risque } \\
\hline - Gain : $2500 \$$ avec $33 \%$ de chances & - Gain : $2400 \$$ avec $34 \%$ de chances \\
- Gain : $\quad 0 \$$ avec $67 \%$ de chances & - Gain : $0 \$$ avec $66 \%$ de chances \\
\hline $83 \%$ des participants ont choisi la loterie C & $17 \%$ des participants ont choisi la loterie D \\
\hline
\end{tabular}

Pour la première configuration, même si la loterie A propose une probabilité conséquente de $99 \%$ de gagner au moins $2400 \$$, et une probabilité bien plus faible de ne rien gagner, la majorité des participants (82\%) ont opté pour la loterie B offrant (à 100\%) un gain certain de $2400 \$$. L'écart de probabilité de $1 \%(100 \%$ - 99\%) a apparemment été dissuasive et a donc fait basculer les préférences des participants. 
Quant à la deuxième configuration, le même niveau de probabilité de $1 \%$ représente l'écart qui sépare les chances de gagner respectivement $2500 \$$ (33\%) pour la loterie C et $2400 \$(34 \%)$ pour la loterie D. Pourtant, la majorité des participants (83\%) ont opté pour la loterie C, même si la probabilité y afférente est inférieure. Dans ce cas, ce sont plutôt les $2500 \$$ qui seraient apparemment attractifs aux yeux des participants par rapport aux $2400 \$$ proposés ailleurs.

La principale conclusion de ce raisonnement consiste à confirmer que les agents ne perçoivent pas exactement de la même manière un même niveau de risque (en l'occurrence 1\%) (Kahneman et Tversky [1979]). C'est ce traitement intuitif des pondérations qui génère ladite déformation des probabilités objectives.

La comptabilité mentale, qui résume les activités d'ordre cognitif qu'un agent réalise pour gérer ses affaires financières, est fortement liée à ce phénomène de déformation des pondérations (Thaler [1999]). Concrètement, les agents divisent le même portefeuille en plusieurs fragments distincts dont chacun est investi pour un objectif particulier (Thaler [1980]).

C'est pourquoi le courant comportementaliste propose de mêler le calcul des probabilités, via une fonction de pondération, au calcul du degré de satisfaction, via une fonction d'utilité (Tversky et al. [1992]).

La question qui se pose ici est la suivante : Sur quelle base devrait-on mesurer la satisfaction ? Un élément de réponse, plutôt fiable et largement utilisé, stipule qu'au lieu du niveau absolu de la richesse, c'est en fonction des fluctuations de cette richesse que le degré de satisfaction doit être mesuré (Kahneman et al. [1979]).

En somme, il ne s'agit pas de ces seules valeurs ajoutées. La finance comportementale apporte davantage d'enseignements, comme développé ci-après.

\section{Apports de la théorie financière comportementale}

Notre inclination pour le présent sujet émane en partie du fait que la communauté scientifique donne progressivement raison à l'introduction de la dimension psychologique dans l'analyse et l'explication des phénomènes liés au marché.

C'est pourquoi nous situons académiquement la place de la finance comportementale, avant de mettre en évidence ses apports.

\subsection{Positionnement académique de la finance comportementale}

Pour résumer, au lendemain de la crise financière de 2008, Shefrin [2009] 1'a dans l'ensemble imputée à des phénomènes psychologiques.

Justement, à travers l'introduction du facteur psychologique, la finance comportementale a progressivement obtenu la reconnaissance de la communauté scientifique à son égard.

\subsubsection{Consécration du courant comportementaliste}

La théorie financière comportementale a non seulement acquis une certaine reconnaissance de la communauté scientifique mondiale, mais a aussi et surtout proposé des travaux de recherche pertinents, décorés ponctuellement par une distinction connue en économie : le Prix Nobel d'économie.

A commencer par le psychologue et économiste Daniel Kahneman, coauteur avec Amos Tversky de l'article précurseur de la finance comportementale, qui a obtenu le Prix Nobel d'économie en 2002, 
pour ses travaux de recherche ayant formellement fondé la théorie des perspectives (Snineh et al. [2021]).

Quinze années plus tard, soit en 2017, ce même Prix a été décerné à leur disciple Richard H. Thaler pour ses travaux sur les mécanismes psychologiques et sociaux mis à l'œuvre dans les décisions des consommateurs ou des investisseurs.

Il convient de remonter aussi dans le temps jusqu'à 1978 pour trouver trace à une autre consécration par le Prix Nobel d'économie, dont le titulaire est Herbert Alexander Simon pour ses travaux de recherches à travers lesquels il a fondé le concept de rationalité limitée chez les agents.

Cette liste d'auteurs n'est pas exhaustive. En tous cas, un point commun se dégage de leurs travaux de recherche: le comportement. L'analyse des comportements humains relève du domaine de la psychologie qui est elle-même une discipline des sciences cognitives.

\subsubsection{Rôle des sciences cognitives}

L'essor de la finance comportementale est le fruit des progrès réalisés par les sciences cognitives (Orléan [1994]). Nées dans les années 1940, les sciences cognitives forment un ensemble de disciplines scientifiques (psychologie, linguistique, philosophie, neuroscience, etc.) dont l'objectif est de comprendre le mécanisme de la pensée humaine.

Dans le cadre de la théorie financière comportementale, le marché financier est appréhendé à travers l'usage de ces sciences cognitives. Concrètement, les investisseurs ne sont plus considérés comme des êtres rationnels et indépendants mais plutôt comme des individus obéissant à l'influence de leurs émotions, et qui représentent nécessairement l'objet de plusieurs biais de raisonnement, surtout lorsqu'ils interagissent entre eux (Thaler [2016]). Avec cet angle de vue nouveau, la finance comportementale apporte des éclairements intéressants aux zones d'ombre laissées par la théorie financière classique.

La finance comportementale suppose ainsi que l'investisseur pris individuellement décide d'investir (ou non) dans une action, en y voyant plusieurs caractéristiques (Nagy et al. [1994], Clark-Murphy et al. [2004]). Pour cela, il suit ses propres préférences (Fama et al. [2007]). C'est pourquoi le processus de décision en la matière semble plus complexe que l'on puisse imaginer. Lesdites préférences sont difficilement définissables (Benartzi et al. [2001]), et concernent des aspects autres que l'enjeu binaire risque-gain (Fisher et al. [1997]).

Par ailleurs, les considérations motivant les choix de l'agent sont en réalité très diverses (Hoffmann [2007]). Ses choix sont souvent biaisés au moins par son niveau cognitif et son éventuelle attitude face au risque (De Bondt [1998]). Il pourrait donc en découler un éloignement du prix de l'actif en question par rapport à sa valeur fondamentale.

Il convient de remonter le temps pour trouver trace à un premier essai dans ce sens. En effet, John Maynard Keynes [1936] a démontré que le cours d'un titre dévie de sa valeur fondamentale suite à la formation d'une opinion collective, ce qui entraîne à moyen et à long termes la volatilité excessive sur le marché. Keynes estime que les agents convergent tout simplement vers une opinion commune quand le marché ne propose pas suffisamment d'opinions alternatives à choisir.

Schindler [2007] et Montier [2008] ont également travaillé sur les biais d'ordre comportemental. Le premier estime que ces biais conditionnent et donc expliquent les circonstances dans lesquelles le marché évolue. Le second démontre leur influence sur le choix des titres. 
Il s'agit là de quelques-uns parmi une longue liste de biais. Pour les étudier, nous nous sommes inspirés, entre autres, d'une référence bibliographique produite par l'économiste américain Hirshleifer [2001]. Il s'agit d'un travail de recherche qui propose pertinemment une cartographie assez large des biais intrinsèques aux agents en interaction.

\subsection{Biais comportementaux}

Dans ce domaine, les travaux de Hirshleifer [2001] sont très riches. Ses contributions sont basées en partie sur les travaux développés par Trivers [1985], fondateur des premiers éléments d'analyse de l'excès de confiance. Ce dernier représente le biais comportemental le plus examiné dans la littérature en la matière.

Trivers [1985] a fondé la théorie de l'auto-déception selon laquelle l'agent tend parfois à mentir, en cachant à ses concurrents certains faits ou certaines informations le concernant. Avec le temps passé dans cet exercice, il finit, sans qu'il le conçoive réellement, par croire à son propre mensonge. À titre d'illustration, s'il exagère en s'attribuant certains points forts qu'il ne possède pas en réalité (facultés intellectuelles fortes, clairvoyance, etc.), il finira par croire qu'il les a vraiment. Par conséquent, ses jugements seront nécessairement biaisés car ils seront en deçà de ce que l'on pourrait attendre de lui.

Il s'agit là d'une parmi plusieurs formes d'erreurs de jugement. Mais, la plus connue est l'excès de confiance qui comporte en son sein plusieurs autres biais.

\subsubsection{Excès de confiance}

Le courant comportementaliste s'accorde à lier la volatilité excessive des cours à l'existence d'agents sur-confiants (Ko et al. [2007]). L'étude du biais d'excès de confiance propose plusieurs lectures qui aident à comprendre certaines origines de la déviation des prix de leurs valeurs fondamentales.

D'abord, l'excès de confiance peut provenir de la chance du débutant, ou tout simplement d'un pari chanceux. Ceux qui croient en la chance continueront de croire à la même stratégie qui les a faits gagner (Darke et al. [1997]).

Ensuite, l'excès de confiance peut également provenir d'agents qui cherchent à appartenir à une élite, ou à une minorité, dans un jeu que la littérature appelle "jeu de minorité" (Arthur [1994]).

En général, un investisseur sur-confiant se trompe quant au degré de précision des connaissances qu'il détient. Il croit donc en la précision de son appréciation et omet de considérer celles que ses concurrents ont. Par conséquent, si les risques relatifs aux actifs qu'il négocie sont sous-estimés, l'agent en question possède alors un portefeuille plus risqué (Hirshleifer et al. [2001]), voire non suffisamment diversifié qu'il ne le pense (Skata [2008]).

En principe, selon la logique élémentaire de la finance, plus le titre est risqué, plus son potentiel de gain est important. Or, devant leurs homologues rationnels similairement averses au risque, les agents surconfiants, possédant des portefeuilles dont le risque provient de ce caractère de sur-confiance, ne peuvent pas se vanter d'un quelconque potentiel de gain auquel ils aspirent à tort (Hirshleifer [2001]).

Parfois même, un marché où évoluent des investisseurs sur-confiants et qui affiche des valeurs très volatiles, profite davantage aux investisseurs supposés rationnels car le calcul que ces derniers font leur permet de repérer et d'éviter ce type de risque fictif lié à l'excès de confiance (Dumas et al. [2007]).

C'est ainsi que Barber et al. [2000] démontrent que la volatilité excessive des valeurs détenues par des agents sur- confiants et le niveau de leur confiance sont positivement corrélés. 
Une conclusion pareille a couronné les travaux de recherches de Gervais et al. [2001] et ceux d'Acker et al. [2008] qui confirment la coexistence quasi-systématique d'agents sur-confiants et de titres excessivement volatiles, quand le nombre de transactions que ces agents réalisent est important.

Benos [1998], lui, a mis en interaction des agents sur-confiants et des investisseurs rationnels conservateurs sur un marché et a remarqué que les cours deviennent plus volatiles. À travers son modèle, il démontre que l'impact de l'excès de confiance chez les premiers sur leur manière de traiter l'information, combiné à celui du caractère conservateur chez les seconds sur leurs options stratégiques, intensifie la volatilité des prix.

Chuang et al. [2006] ont par ailleurs démontré que le contenu de l'information traitée a un impact sur la volatilité des cours. Ce contenu est de deux types selon Slovic [1973] :

Contenu brut: il s'agit d'informations relatives à des faits, généralement officielles comme celles émanant des sociétés de Bourse et des sociétés de gestion, ou celles publiées par le régulateur du marché : notes, circulaires, révision des seuils de variation maximale, etc. C'est ce type d'information qui sert objectivement de base d'aide à la décision.

Contenu traité : il s'agit d'analyses non nécessairement officielles, faites par les banques d'affaires ou les experts, ou d'avis fondés, formulés par les intermédiaires, ou encore d'études spécialisées dûment indexées. Ce type d'information sert uniquement à améliorer le processus de décision chez l'investisseur.

L'on parle également de biais d'excès de confiance quand le fait d'accéder le premier à un maximum d'informations sur le marché devient l'objet de compétition. L'expérimentation menée par Slovic [1973] confirme que ce n'est pas le volume d'informations détenues qui importe mais plutôt l'usage que l'agent peut en faire. En effet, contrairement à ce que l'on peut penser, plus l'investisseur détient d'informations plus ses prédictions sont moins précises et peu pertinentes. Outre la sur-confiance qu'il acquiert en conséquence, il aura beaucoup de mal à les traiter simultanément. En plus, son excès de confiance et ses prédictions trempeuses l'amènent à miser davantage, ce qui représente un coût de plus. (Slovic [1973])

L'excès de confiance peut également provenir de la "qualité" de l'investisseur. En effet, les professionnels sont sur-confiants plus que les autres agents, car ils ont tendance à croire qu'ils sont plus clairvoyants en anticipant les bonnes opportunités (Griffin et al. [1992]).

Dans ce même cadre, les agents sur-confiants ont tendance à surestimer la pertinence et donc les poids des informations privées qu'ils détiennent, ce qui entraîne la volatilité des prix (Daniel et al. [1998]). D'autant que l'avènement des informations publiques, considérées comme moins fiables, ne corrige que partiellement la déviation des cours (Daniel et al. [2001]).

Par ailleurs, la sur-confiance peut pousser les investisseurs à croire que les rentabilités antérieurement réalisées sur le marché reviennent à leurs seules compétences intellectuelles et techniques (Glaser et al. [2009]). Il en découle une revalorisation des cours tirant à la hausse les autres actifs objet de négociation.

Cette forme de croyance résume un biais dit d'attribution. Selon ce biais, l'agent s'approprie les origines des placements gagnants, tandis qu'il impute toute perte à la malchance (Lau et al. [1980]), voire à des facteurs externes (Daniel et al. [1998]). Par conséquent, l'agent n'apprend quasiment pas et renonce à ajuster ses méthodes.

Enfin, l'excès de confiance peut facilement mener à un excès d'optimisme. Il s'agit d'une attitude subjective qui peut pousser l'investisseur à surestimer la probabilité de gain ou à sous-estimer celle 
relative à la perte (De Bondt et al. [1990]), avec les conséquences sur la formation des prix que l'on connait.

L'excès d'optimisme peut quant à lui générer l'effet momentum selon lequel les rendements positifs enregistrés par un titre pendant une période de court terme tendent à se produire lors de la période suivante (Jegadeesh et al. [1993]). Il en est de même pour les contreperformances du titre. Les agents achètent alors le titre dans le premier cas et s'en débarrassent dans le second (Griffin et al. [2003]).

L'excès de confiance étant traité, il convient maintenant d'examiner les autres biais et heuristiques d'ordre cognitif.

\subsubsection{Heuristiques, émotions et biais cognitifs}

L'analyse de l'aspect comportemental chez les investisseurs révèle un certain nombre d'erreurs d'appréciation suite à plusieurs biais et heuristiques (Legros et al. [2011]). En plus de l'excès de confiance, ces derniers contribuent aux mauvaises performances (Glaser et al. [2007]), et poussent à des prises de risque excessives (Barber et al. [2001]).

\section{Heuristiques}

Les heuristiques sont des sortes de raccourcis que le cerveau utilise lors de la prise de décision. Ces simplifications sont en général erronées pour une raison simple: l'imprévoyance individuelle d'un agent fait que ses capacités en termes de concentration, de mémorisation, de calcul mental, etc., ne sont pas sans limites (Szyszka [2013]). C'est pourquoi l'individu, face à une situation d'incertitude, recourt à son intuition pour trouver un élément de réponse simplifié, en peu de temps et avec moins d'effort (Hirshleifer [2001]).

Cette attitude simpliste a une première forme, appelée biais d'ancrage, selon laquelle la première impression contribue largement à la structuration du raisonnement qui la suit (Hirshleifer [2001]).

Le biais de représentativité fait également partie de ces erreurs de raisonnement. Face à un risque, la représentation chez l'individu le pousse à adopter une approche fondée sur des faits passés similaires plutôt que sur des calculs probabilistes ou statistiques (Tversky et al. [1974]). L'agent se facilite donc la tâche en rapprochant la situation à laquelle il est confrontée à la réalité qui lui est familière.

Ce biais peut aussi procurer à l'individu la conviction que son modèle prédictif est fiable voire infaillible, et ignorer que la survenance de l'événement désiré n'est pas certaine et n'a qu'une probabilité d'occurrence susceptible d'être faible.

Selon cette même heuristique, la volatilité des cours peut également être le résultat des réactions exagérées des agents pour lesquels un titre, constamment valorisé, garde toujours des perspectives de rentabilité positive (De Bondt et al. [1985]).

La loi des petits nombres peut apporter un enseignement intéressant sur ce point. Les investisseurs ont tendance à surestimer la capacité des petits échantillons, en l'occurrence une courte séquence d'observations, à refléter les attributs d'une valeur cotée depuis longtemps (Rabin [2002]).

À titre d'exemple, quand les dividendes distribués en fin d'année connaissent une augmentation, cela ne veut pas dire que le taux de croissance moyen y afférent a augmenté. Si les investisseurs croient que c'est le cas, leurs comportements peuvent tirer les prix vers la hausse, entraînant la volatilité des cours (Barberis et al. [2002]). 


\section{Émotions non maitrisables}

De nombreuses études démontrent que plusieurs biais issus des émotions impactent le processus de prise de décision chez l'être humain. D'autant plus que maitriser le côté émotionnel n'est pas une certitude.

La perception du risque et l'attitude face à sa réalisation dépendent du tempérament du moment de l'investisseur concerné. Si ce dernier est de bonne humeur, il est plutôt optimiste. Autrement, son mauvais tempérament le pousserait au pessimisme et nourrirait chez lui un certain esprit critique (Wright et al. [1992]).

Il s'agit là d'une réalité vérifiable. Si c'est le cas, qu'est-ce que l'investisseur peut-il faire alors ? Une première réaction peut consister à essayer de maîtriser ses émotions.

S'autocontrôler, ou maîtriser ses émotions, est une attitude compliquée à atteindre. Conscient de l'évidence de ce postulat, l'agent a tendance à privilégier un horizon temporel de court terme pour des rentabilités relativement moyennes, plutôt que d'investir à long terme pour des niveaux de gains plus élevés (Baumeister et al. [1998]). Or, c'est contraire à la logique élémentaire de l'investissement en Bourse.

L'investisseur opte par ailleurs pour la stratégie qui, en cas de perte, lui procurerait le moins de sentiments de regret (Loomes et al. [1982]). Le désir d'éviter ce regret peut le pousser à agir dans la disproportion (Barber et al. [2001]).

En outre, face au risque inhérent à l'investissement, l'agent éprouve une certaine peur de l'inconnu. Il peut par conséquent prendre des décisions non raisonnables, voire paradoxales (Ellsberg [1961]).

\section{Biais cognitifs}

Tout processus de prise de décision comporte nécessairement une étape de traitement de l'information. Cette étape est souvent complexe, c'est pourquoi une décision est généralement difficile à prendre.

En effet, sur un marché, l'abondance de l'information et la multitude de ses sources peuvent créer une désinformation ou un bruit de fond. En plus, l'existence de biais cognitifs (Kahneman et al. [1979]), dits aussi informationnels, peut empêcher l'individu d'exploiter convenablement l'information.

Une décision biaisée peut donc trouver son explication dans l'un des biais liés à l'information.

Le premier biais informationnel est dit biais rétrospectif. Selon ce biais, une fois l'information perçue par l'investisseur, ce dernier tend à croire qu'il l'a toujours sue (Kahneman et al. [1979]).

Le deuxième est dit biais de confirmation. Selon ce biais, l'agent a tendance à ne tenir en compte que les informations qui confirment son raisonnement et son appréciation. Les informations qui les infirment sont donc ignorées (Wason [1960], Grobman [2018]).

Aussi simple qu'il puisse paraitre, le biais de confirmation est particulièrement perturbateur. En effet, si avant l'ouverture d'une séance de Bourse, l'investisseur reçoit des informations confirmant majoritairement la décision d'investissement qu'il envisage de prendre, il est normal qu'il ait tendance à ignorer les nouvelles informations. Même si ces dernières peuvent porter des enseignements sur un risque potentiel à venir, il s'abstiendra à ajuster son appréciation.

Parallèlement à ce raisonnement, le biais d'attribution est un raccourci qui pousse l'agent -à tort- à attribuer des pondérations surestimées aux informations qui confirment ses croyances, au détriment des 
autres données disponibles sur le marché et auxquelles il alloue des poids sous-estimés (Barberis et al. [1998]).

Par ailleurs, le biais mimétique ou de suivisme, tel qu'il est fondé initialement par Keynes [1936], stipule que les agents incertains quant aux fondamentaux de la finance recourent à l'opinion des autres agents pour trouver une information pertinente relative aux actifs mis en jeu. Ce comportement peut être mutuel lorsque les investisseurs sont reliés par un lien d'amitié (Sornette [2003]). Cette attitude, très répandue sur le marché, conduit à la formation d'une opinion collective (Orléan [2000]).

La volatilité excessive sur le marché en est une conséquence, car cette forme d'unanimité neutralise la connexion qui lie les cours à leur valeur fondamentale. Celle-ci est remplacée par l'opinion moyenne du marché (Cajueiro et al. [2009]).

Jondeau [2001] a travaillé sur les origines d'un tel comportement mimétique et montre que des agents tendent à imiter les investisseurs disposant d'informations privilégiées sur un actif. Si une cohorte d'agents les imite pour la gratuité de cet acte, d'autres le font juste pour s'y aligner et ne pas contredire (Graham [1999]).

Quoiqu'il en soit, le fait pour un agent $\mathrm{A}$ de copier auprès d'un agent $\mathrm{B}$ n'est pas systématiquement néfaste en soi. L'agent B peut par exemple être un expert en finance dont la pertinence des connaissances est reconnue.

Mais, dépourvu d'analyse subjective, le comportement suiviste amplifie non seulement la volatilité des valeurs (Tan et al. [2008]) mais aussi les volumes des transactions (Capelle-Blancard [2018]).

Rappelons que l'opinion collective est une sorte d'agrégation d'informations. Alors, quand les agents s'imitent, ce processus d'agrégation peut échouer et donc contribuer à l'amplification de la volatilité des cours, surtout lorsque les opérations se font par séquences (Cipriani et al. [2008]).

Le problème se pose également quand le copiage se répète et se généralise, parce que cela peut entraîner la création des bulles spéculatives.

\section{Conclusion}

Il n'est plus à démontrer aujourd'hui que le comportement rationnel n'est ni une attitude toujours observée ni une caractéristique intrinsèque chez tous les agents constituant le marché. Il en est de même pour l'existence de biais comportementaux de plusieurs ordres affectant les décisions des investisseurs dans de larges proportions.

Et si la communauté scientifique a fini par reconnaitre l'apport de la finance comportementale, c'est parce que celle-ci est fondée sur plusieurs expérimentations solides, puisant dans les résultats des recherches en sciences cognitives, rendant les modèles qui en sont issus plus proches de la réalité.

Il en découle des théories nouvelles, orientées vers les perspectives relatives à l'investissement, plutôt que vers l'utilité que ce dernier peut procurer.

\section{BIBLIOGRAPHIE}

[1] ACKER D. and DUCK N.W. [2008], "Cross-cultural overconfidence and biased self-attribution", The Journal of Socio-Economics, 37, pp. 1815-1824.

[2] ALLAIS M. [1953], "Le Comportement de l'Homme Rationnel devant le Risque: Critique des Postulats et Axiomes de l'Ecole Americaine", Econometrica, 21(4), 503-546.

[3] ARTHUR W.B. [1994], "Inductive reasoning and bounded rationality (The El Farol Problem)", American Economic Review, 84, 406-411. 
[4] BARBER B.M and ODEAN T. [2000], "Trading Is Hazardous to Your Wealth: The Common Stock Investment Performance of Individual Investors", The Journal of Finance, 55, 773-806.

[5] BARBER B.M and ODEAN T. [2001], "Boys Will Be Boys: Gender, Overconfidence, And Common Stock Investment", Quarterly Journal of Economics 116(1):261-292.

[6] BARBERIS N. and HUANG M. [2001], "Mental Accounting, Loss Aversion, and Individual Stock Returns", The Journal of Finance. 56. 1247 - 1292.

[7] BARBERIS N. and THALER R. [2002], "A survey of behavioral finance", National Bureau of Economic Research Working Paper Series, No. 9222.

[8] BARBERIS N. and THALER R. [2002], "A survey of behavioral finance", National Bureau of Economic Research Working Paper Series, No. 9222.

[9] BARBERIS N., HUANG M. and SANTOS T. [2001], "Prospect Theory And Asset Prices" Quarterly Journal of Economics, Oxford University Press, Volume $116 \mathrm{n}^{\circ}$ 1, pp 1-53.

[10] BARBERIS N., SHLEIFER A. and VISHNY R. [1998], "A Model of Investor Sentiment", Journal of Financial Economics 49 (3): 307-343.

[11] BAUMEISTER R. F., BRATSLAVSKY E., MURAVEN M. and TICE D. M. [1998], "Ego depletion: Is the active self a limited resource?", Journal of Personality and Social Psychology, 74(5), 1252-1265.

[12] BENARTZI S. [2001], "Excessive Extrapolation and the Allocation of 401(k) Accounts to Company Stock", The Journal of Finance, 56.

[13] BENARTZI S. and THALER R. H. [2001], "Naive Diversification Strategies in Defined Contribution Saving Plans". The American Economic Review, 91(1), 79-98.

[14] BENOS A.V. [1998], "Aggressiveness and survival of overconfident traders", Journal of Financial Markets, 1, pp. 353-383.

[15] BERK J. and DeMARZO P. [2017], "Corporate finance", 4th Global Edition.

[16] CAJUEIRO D.O. and TABAK B.M. [2009], "Multifractality and herding behavior in the Japanese stock market, Chaos, Solitons \& Fractals", Volume 40, Issue 1, Pages 497-504.

[17] CAPELLE-BLANCARD G. [2018], "What is the point of (the hundreds of thousands of billions of) stock transactions?", Comparative Economic Studies, 60(1), 15-33.

[18] CHUANG W.I. and LEE B.S. [2006], "An empirical evaluation of the overconfidence hypothesis", Journal of Banking \& Finance, 30, pp. 2489-2515.

[19] CIPRIANI M. and GUARINO A. [2008], "Herd Behavior and Contagion in Financial Markets", The B.E. Journal of Theoretical Economics, 8, issue 1, p. 1-56.

[20] CLARK-MURPHY M. and SOUTAR G.N. [2004], "What individual investors value: some Australian evidence", Journal of Economic Psychology, vol. 25, n 4 .

[21] COOPER M., DIMITROV O. and RAU P., [2001]. "A Rose.com by Any Other Name," Journal of Finance, American Finance Association, vol. 56(6), pages 2371-2388.

[22] D'AVOLIO G. [2002], "The Market for Borrowing Stock", Journal of Financial Economics. 66. 271-306.

[23] DANIEL K., HIRSHLEIFER D. and SUBRAHMANYAM A. [1998], "Investor Psychology and Security Market under- and Overreactions", The Journal of Finance, 53(6), 1839-1885.

[24] DANIEL K., HIRSHLEIFER D. and SUBRAHMANYAM A. [2001], "Overconfidence, Arbitrage, and Equilibrium Asset Pricing", The Journal of Finance, 56(3), 921-965.

[25] DARKE P.R. and FREEDMAN J.L. [1997], "The Belief in Good Luck Scale", JOURNAL OF RESEARCH IN PERSONALITY 31, 486-511,

[26] DE BONDT W. and THALER R. [1985], "Does the Stock Market Overreact?", The Journal of Finance, 40(3), 793-805.

[27] DE BONDT W. F. M. and THALER R. H. [1990], "Do Security analysts overreact ?", American Economic Review, 80(2), p. 52-57.

[28] De BONDT W. F.M. [1998], "A portrait of the individual investor", European Economic Review, 42. 
[29] DE BONDT W., SHEFRIN H., MURADOGLU G. and STAIKOURAS S. [2008], "Behavioural Finance: Quo Vadis?", Journal of Applied Finance. Volume 18.

[30] De LONG J., SHLEIFER A., SUMMERS L. and WALDMANN R. [1990], "Noise Trader Risk in Financial Markets", Journal of Political Economy, 98(4), 703-738.

[31] DUMAS B., KURSHEV A. and UPPAL R. [2007], "Equilibrium portfolio strategies in the presence of sentiment risk and excess volatility", Swiss Finance Institute, 7, pp. 1-49.

[32] ELLSBERG D. [1961], "Risk, Ambiguity, and the Savage Axioms", The Quarterly Journal of Economics, 75(4), 643-669.

[33] FAMA E. [1965], "The Behavior of Stock-Market Prices", Journal of Business, vol. 38, n 1, p. 31-105.

[34] FAMA E.F. and FRENCH K.R. [2007], "The anatomy of value and growth stock returns", Financial Analysts Journal 63(6), 44-54.

[35] FISHER K. and STATMAN M. [1997], "Investment advice from mutual fund companies", The Journal of Portfolio Management, 24 (1) 9-25.

[36] FRIEDMAN M. [1953], "The case for flexible exchange rates", in 'Essays in positive economics', University of Chicago Press, Chicago, IL.

[37] FRIEDMAN M. and SAVAGE L. [1948], "The Utility Analysis of Choices Involving Risk", Journal of Political Economy, 56(4), 279-304.

[38] GERVAIS S. and ODEAN T. [2001],"Learning to Be Overconfident", The Review of Financial Studies, 14(1), $1-27$.

[39] GLASER M. and WEBER M. [2007], "Overconfidence and trading volume", The Geneva Risk and Insurance Review, 32(1), 1-36.

[40] GLASER M. and WEBER M. [2009], "Which Past Returns Affect Trading Volume?", Journal of Financial Markets, Vol. 12, pp. 1-31.

[41] GOETZMAN W. and KUMAR A. [2008],"Equity Portfolio Diversification", Review of Finance, Volume 12, Issue 3, Pages 433-463.

[42] GRAHAM J.R. [1999], "Herding among Investment Newsletters: Theory and Evidence », Journal of Finance, 4, 237-268.

[43] GRIFFIN D. and TVERSKY A. [1992], "The weighing of evidence and the determinants of confidence", Cognitive Psychology, Volume 24, Issue 3, Pages 411-435.

[44] GRIFFIN J.M., XIUQING J. and MARTIN J.S. [2003], "Momentum Investing and Business Cycle Risk: Evidence from Pole to Pole", The Journal of Finance, 58(6), 2515-2547.

[45] GRINBLATT M. and KELOHARJU M. [2000], "The investment behavior and performance of various investor types: a study of Finland's unique data set", Journal of Financial Economics Volume 55, Issue 1, Pages 43-67.

[46] GROBMAN K.H [2018], "Confirmation bias and the 2-4-6 game: How do we test our perspectives?", In R. L. Miller (Ed.) Promoting Psychological Science: A Compendium of Laboratory Exercises for Teachers of High School Psychology, (pp. 286-290), Washington, DC: American Psychological Association Society for the Teaching of Psychology.

[47] GROSSMAN S. J. and SHILLER R. [1981], "The Determinants of the Variability of Stock Market Prices", The American Economic Review, 71(2), 222-227.

[48] HIRSHLEIFER D. and LUO G. [2001], "On the survival of overconfident traders in a competitive securities market", Journal of Financial Markets, vol. 4, issue 1, 73-84.

[49] HIRSHLEIFER, D. [2001], "Investor Psychology and Asset Pricing", The Journal of Finance, Volume 56, Issue 4 p. $1533-1597$.

[50] HOFFMANN W. H. [2007], "Strategies for managing a portfolio of alliances", Strategic Management Journal Volume 28, Issue 8 p. 827-856.

[51] HOMMES C., SONNEMANS J., TUINSTRA J. and VAN DE VELDEN H. [2008], "Expectations and bubbles in asset pricing experiments", Journal of Economic Behavior \& Organization, 67, pp. 116-133.

[52] HUBERMAN G. [2001], "Familiarity Breeds Investment", Review of Financial Studies 14, 659-680. 
[53] IBBOSTON R. G. and JAFFE J. F. [1975], "'Hot Issue" Markets", The Journal of Finance, 30(4), 1027-1042.

[54] JEGADEESH N. and TITMAN S. [1993], "Returns to Buying Winners and Selling Losers: Implications for Stock Market Efficiency", The Journal of Finance, 48(1), 65-91.

[55] JONDEAU E. [2001], "Le comportement mimétique sur les marchés de capitaux", BULLETIN DE LA BANQUE DE FRANCE - $\mathrm{N}^{\circ}$ 95, Direction générale des Études et des Relations internationales, Centre de recherche.

[56] KAHNEMAN D. and TVERSKY A. [1979], "Prospect theory: an analysis of decision under risk", Econometrica, Volume 47, Number 2.

[57] KAHNEMAN D., SLOVIC P., and TVERSKY A. [1982], "Judgment under Uncertainty: Heuristics and Biases", Cambridge, Cambridge University Press.

[58] KEYNES J. M. [1936], "The General Theory of Employment, Interest, and Money", Hacourt, Brace \& World, New York.

[59] KO K.J. and HUANG Z. [2007], "Arrogance can be a virtue: overconfidence, information acquisition, and market efficiency", Journal of Financial Economics, Volume 84, Issue 2, Pages 529-560.

[60] LAMONT O. A., et THALER R. H. [2003], "Anomalies: The law of one price in financial market", Journal of Economic Perspectives, 17(4), p. 191-202.

[61] LARDIC S. et MIGNON V. [2006], "L’efficience informationnelle des marchés financiers", Éditions La Découverte, Paris.

[62] LAU R. and RUSSELL D. [1980], "Attributions in the Sports Pages", Journal of Personality and Social Psychology 39(1):29-38.

[63] LEGER J-Y. [2008], "La communication financière", Editions Dunod, Collection Fonctions de l'entreprise.

[64] LEGROS F., BOURROUSSE H. and SOULAT L. [2011], "Le comportement des investisseurs institutionnels relève-t-il de la finance comportementale ?", Institut Louis Bachelier, Paris .

[65] LEROY S. and PORTER R. [1981], "The Present-Value Relation: Tests Based on Implied Variance Bounds", Econometrica, 49(3), 555-574.

[66] LOOMES G. and SUGDEN R. [1982], "Regret Theory: An Alternative Theory of Rational Choice Under Uncertainty", The Economic Journal, 92(368), 805-824.

[67] MARKOWITZ H. [1952], "Portfolio Selection", The Journal of Finance, vol. 7, p. 77-91.

[68] MERTON R. [1987], "A Simple Model of Capital Market Equilibrium with Incomplete Information", Journal of Finance, vol. 42, issue 3, 483-510.

[69] MILlER M. H. and MODIGLIANI F. [1961], "Dividend Policy, Growth, and the Valuation of Shares". The Journal of Business, 34(4), 411-433.

[70] MONTIER J. [2008], "Behavioural Finance, Insights into Irrationnal Minds and Markets", John Wiley \& Sons.

[71] NAGY R. A. and Obenberger R. W. [1994], « Factors influencing individual investor behavior », Financial Analysts Journal, vol. 50, n4, pp. 63-68.

[72] ORLEAN A. [1994], "Analyse des phénomènes d'influence : de la psychologie sociale et cognitive à l'économie financière", Revue économique. Volume $45, \mathrm{n}^{\circ} 3$.

[73] ORLEAN A. [2000], "L’individu, le marché et l'opinion : réflexions sur le capitalisme financier", Esprit (1940-), 269 (11), 51-75.

[74] ORLEAN A. [2004], « Les marchés financiers sont-ils rationnels ? », Reflets et Perspectives, XLIII / 2, pp. 3541.

[75] ORLEAN A. [2008], "Efficience informationnelle versus Finance comportementale: éléments pour un débat", Axes de la recherche en sciences économiques, Presses de la Rue d’Ulm, pp.1-13.

[76] POLLIN J-P. [2007], "Finance comportementale et volatilité", Université d'Orléans.

[77] RABIN M. [2002], "Inference by Believers in the Law of Small Numbers", The Quarterly Journal of Economics, Volume 117, Issue 3, Pages 775-816.

[78] RUBINSTEIN M. [2000], "Rational market: Yes or no? The affirmative case", Haas School of Business, University of California, Berkeley. 
[79] SCHINDLER M. [2007], "Rumors in Financial Markets: Insights into Behavioral Finance", John Wiley \& Sons.

[80] SCHMIDT C. [2006], "Psychologie cognitive et analyse des décisions économiques", Revue française d'économie, Volume $20 \mathrm{~N}^{\circ} 3$.

[81] SEN A. [1991], "Ethique et finance", Revue d'économie financière, Université de Harvard, Etats-Unis.

[82] SHARPE W. [1964], "Capital Asset Prices : a Theory of Market Equilibrium under Conditions of Risk", Journal of Finance, 19, p. 425-442.

[83] SHARPE W. and ALEXANDER G. [1990], "Investments", Englewood Cliffs, N.J. : Prentice Hall.

[84] SHEFRIN H. [2009], "How Psychological Pitfalls Generated the Global Financial Crisis", VOICES OF WISDOM: UNDERSTANDING THE GLOBAL FINANCIAL CRISIS, Laurence B. Siegel, ed., Research Foundation of CFA Institute.

[85] SHEFRIN H. and STATMAN M. [2000], "Behavioral Portfolio Theory". The Journal of Financial and Quantitative Analysis, 35(2), 127-151.

[86] SHILLER R. [1981], "Do Stock Prices Move Too Much to be Justified by Subsequent Changes in Dividends?", The American Economic Review, 71(3), 421-436.

[87] SHILLER R. [1984], "Stock Prices and Social Dynamics", No 719R, Cowles Foundation Discussion Papers, Cowles Foundation for Research in Economics, Yale University.

[88] SHILLER R. [2003], "From Efficient Markets Theory to Behavioral Finance" Journal of Economic Perspectives, 17 (1): 83-104.

[89] SHLEIFER A. [1986], "Do Demand Curves for Stocks Slope Down?", The Journal of Finance, Volume 41, Issue 3, p. $579-590$.

[90] SHLEIFER A. [2000], "Inefficient Markets, An introduction to Behavioral Finance", Oxford University Press, New York, [2000]

[91] SHLEIFER A. and VISHNY R. W. [1997], "The Limits of Arbitrage". The Journal of Finance, 52(1), 35-55.

[92] SKALA D. [2008], "Overconfidence in psychology and finance: an interdisciplinary literature review", Financial Markets and Institutions, pp.33-50.

[93] SLOVIC P. [1973], "Behavioral Problems of Adhering to a Decision Policy", Institute for Quantitative Research in Finance, California

[94] SMITH A. [1759], "The Theory of Moral Sentiments", repris par Bonar, J. (1926), Philosophy, 1(3), p. 333-353.

[95] SNINEH, M. H., \& MESK, H. (2021). Les théories fondatrices de la finance comportementale. International Journal of Financial Accountability, Economics, Management, and Auditing (IJFAEMA), 3(5), 962-972. https://doi.org/10.52502/ijfaema.v3i5.168

[96] SORNETTE D. [2003], "Why Stock Markets Crash: Critical Events in Complex Financial Systems", Princeton U. Press, Princeton, N.J., (421 pp.).

[97] STIGLITZ J. E. and GROSSMAN S. J. [1980] "On the Impossibility of Informationally Efficient Markets", The American Economic Review, 70(3), 393-408.

[98] STRACCA L.[2004], "Behavioral finance and asset prices: Where do we stand ?", Journal of Economic Psychology, 2, pp. 373-405.

[99] SZYSZKA A. [2013], "Behavioral Finance and capital markets, How investor psychology influences asset princing", Palgrave MacMillan, United States, First Edition.

[100]TAN L., CHIANG T., MASON J. and NELLING E. [2008], "Herding Behavior in Chinese Stock Markets: An Examination of A and B Shares", Pacific-Basin Finance Journal, 16. 61-77.

[101]THALER R. [2005], "Advances in behavioral finance", Vol. II, Russell Sage Foundation \& Princeton University Press.

[102] THALER R. H., [2016], "Behavioral Economics: Past, Present, and Future", American Economic Review, 106 (7), p. 1577-1600.

[103]THALER R.H. [1980], "Toward a positive theory of consumer choice" Journal of Economic Behavior \& Organization, Volume 1, Issue 1, Pages 39-60.

[104]THALER R.H. [1999], "Mental Accounting Matters", Journal of Behavioral Decision Making, 12, 183. 
[105]TRIVERS R. [1985], "Social Evolution", Benjamin/Cummings Publishing Company, 462 pages.

[106]TVERSKY A. and KAHNEMAN D. [1974], "Judgment under Uncertainty: Heuristics and Biases", Science, New Series, Vol. 185, No. 4157, pp. 1124-1131.

[107]TVERSKY A. et KAHNEMAN D. [1992], "Progrès dans la théorie de perspective: représentation cumulative de l'incertitude". Journal of Risk and Uncertainty. 5 (4): 297-323.

[108]WASON P.C. [1960] "On the failure to eliminate hypotheses in a conceptual task", The Quarterly Journal of Experimental Psychology, 12, 129-140.

[109]WRIGHT W. and BOWER G. [1992], "Mood Effects on Subjective Probability Assessment", Organizational Behavior and Human Decision Processes. 52(2):276-291. 